\title{
A REORIENTAÇÃO DO ENSINO E DA PRÁTICA EM ENFERMAGEM: implantação do Pró-Saúde em M ossoró, Brasila
}

\author{
Fátima Raquel Rosado M ORA IS ${ }^{b}$, Ilana D eyse Rocha LEITEc, \\ L ucineire L opes de OLIVEIRA ${ }^{d}$, Renata M eira VERÁS
}

\section{RESUMO}

A F aculdade de Enfermagem da U niversidade do Estado do Rio Grande do N orte, através do Programa Nacional de Reorientação da Formação Profissional em Saúde (Pró-Saúde) visa articular ensino/ serviço contribuindo na reflexão da formação em enfermagem em M ossoró, Rio G rande do N orte. Esta pesquisa objetivou conhecer a implantação e inserção do Pró-Saúde nas U nidades Básicas de Saúde do município de M ossoró, para entender como os enfer meiros se posicionam diante da articulação ensino/ serviço. Foram realizadas entrevistas com seis profissionais que atuam nas U nidades de Saúde parceiras neste projeto. Os resultados demonstraram que estes trabalhadores detinham poucos conhecimentos acerca do Pró-Saúde. Essa falta de conhecimento, associada às dificuldades na integração ensino/ serviço, não contribuía para a reflexão e reorientação das práticas de enfermagem. Portanto, é necessário repensar saberes e práticas, pactuando mudanças que contribuam com uma formação em saúde que estabel eça um cuidado de qual idade, baseado nos princípios estabelecidos pelo Sistema Ú nico de Saúde.

D escritores: Ensino. For mação de recursos humanos. Instituiç̧̃es de saúde, recur sos humanos e serviços. Recursos humanos de enfer magem.

\section{RESUMEN}

L a Facultad de Enfermería de la U niversidad del E stado del Rio G rande do N orte, a través del Programa $\mathrm{N}$ acional de $R$ eorientación de la $F$ ormación P rofesional en Salud ( $P$ ró-S Suúde) articula enseñanza/ servicio contribuyendo en la reflexión de la for mación en enfermería en M ossoró, R io G rande do N or te, B rasil. E sta investigación buscó conocer la implantación e inserción del P ró-Saúde en las U nidades Básicas deSalud del municipio de M ossoró, para entender el posicionamiento delos enfermeros en la articulación enseñanza/ servicio. F ueron realizadas entrevistas con seis profesionales que actúan en las U nidades de Salud colaboradoras en este proyecto. L os resultados demuestran que estos trabajadores poco conocían el P róSaúde. E stefactor, asociado a dificultades en la integración enseñanza/ servicio, dificultaba la reflexión y reorientación delas prácticas de enfer mería. A sí, hay que repensar conocimientos y prácticas, pactando cambi os que contribuyan con una formación en salud que establezca un cuidado de calidad, basado en los principios del Sistema Ú nico de Salud.

D escriptores: E nseñanza. For mación de recursos humanos. Instalaciones para atención de salud, recur sos humanos y servicios. P ersonal de enfermería.

T ítulo: $L$ a reorientación de la enseñanza y práctica de la enfermería: implantación del P ró-Saúde en M ossoró, Brasil.

\section{ABST RACT}

The N ursing School of U niversidade do E stado do R io G rande do N ort,e through the Programa N acional de R eorientação da F ormação P rofissional em Saúde (P ro-H ealth) seeks to articulate teaching and service, contributing to the development of the concept of nursing education in M ossoró, Rio G rande do $\mathrm{N}$ orte, B razil. This research aims to investigate the implementation of $\mathrm{PrOH}$ ealth in the $\mathrm{B}$ asic $\mathrm{U}$ nits of $\mathrm{H}$ ealth in $\mathrm{M}$ ossoró understanding the position of nursing workers in the teaching-service articulation. Interviews were conducted with six nurses who work in health clinics that coolaborated to this project. T he results demonstrated that these w orkers lacked knowledge about the P ro- $\mathrm{H}$ ealth. T his lack of know ledge associated with difficulties in integrating teaching and service did not contribute to the reflection about and reorientation of nursing practice. It is necessary to rethink the knowledge and nursing practices for a gradual change, contributing to improve health care quality, based on the norms established by the U nified H ealth System in B razil.

D escriptors: T eaching. $H$ uman resources formation. $H$ ealth care facilities, manpow er, and services. $N$ ursing staff. T itle: T eaching and nursing practices reorientation: $\mathrm{P}$ ro-H ealth implantation in M ossoró, B razil.

\footnotetext{
${ }^{a}$ Artigo originado do trabalho de conclusão do Curso de Enfermagem apresentado em 2010 à Faculdade de Enfermagem da Universidade do Estado do Rio G rande do N orte (UERN).

b D outora em Psicologia Social, D ocente do Departamento de Enfermagem da UER N, M ossoró, Rio Grande do N orte, Brasil.

' E nfermeira Assistencial da A ssociação de Assistência e Proteção a M aternidade e a Infância de M ossoró, Rio G rande do N orte, Brasil.

d E nfermeira, D outoranda pel o Programa de Pós-G raduação em Ciências Sociais da U niver sidade Feder al do R io G rande do N orte (U F R N ), Docente do Departamento de Enfermagem da UERN, M ossoró, Rio Grande do N orte, Brasil.

e D outora em Psicologia Social, D ocente do Bacharelado Interdisciplinar da U niversidade Federal da Bahia (U F BA ), Salvador, Bahia, Brasil.
} 


\section{INT RODUÇÃO}

0 processo saúde/ doença apresenta-se como dinâmico, e susceptível às situações que ocorrem nos difer entes contextos de vida em sociedade. N essa perspectiva, as instituições responsáveis pela for mação em saúde devem estabelecer estruturas curriculares que dêem conta das diferentes necessidades biológicas, econômicas, culturais e sociais da população.

Todavia, a formação em saúde ainda tende a seguir uma lógica linear e parcelar, numa perspectiva tradicional, que tende a trabalhar domínios de conhecimentos mantendo posturas práticas já arraigadas ${ }^{(1)}$. N essa organização do processo ensino/ aprendizagem há uma tendência a construção de um pensar/fazer focado nas necessidades biológicas que se expressam no corpo das pessoas que frequentam os espaços assistenciais. Esta produção do conhecimento acaba estimulando a compreensão do doente enquanto um órgão e/ ou patologia, tornando as pessoas capazes em lidar com as partes em separado e incapazes em compreender e trabalhar com o todo (2). Assim, são desenvolvidas ações imediatistas, com os recursos tecnológicos disponíveis e focadas na perspectiva de diagnosticar e curar a doença física.

Este modo de pensar a saúde culminou com a constituição de uma "colcha de retalhos", desarticulando corpos, almas, natureza e sociedade. Os indivíduos não são percebidos como seres sociais, envolvidos por um contexto, o que afeta à sua forma de estar no mundo e, por consequência, a sua saúde ${ }^{(3)}$. A lém disso, este modelo de formação não conseguiu conformar uma cobertura de qualidade, coerente com os princípios de integralidade, equidade, univer salidade e participação comunitária proposto pelo Sistema Ú nico de Saúde $(\mathrm{SU} S)^{(4)}$.

Diante destes aspectos, o processo ensino/ aprendizagem em saúde, nos últimos anos, tem sido alvo de reflexões por não ter conseguido interpretar e/ ou modificar o processo saúde/ doença nos diferentes espaços. A I guns apontam a educação e as necessidades de mudança na formação como o nó crítico para as reais transformações na organização das práticas em saúde ${ }^{(1)}$. N a atualidade, as políticas de educação/ saúde vigentes tentam estimular a formação com eixo na integralidade para substituir o modelo tradicional da organização do cuidado ao ser humano(5).
Entre as políticas com este propósito, é possível citar o Programa Nacional de Reorientação da Formação Profissional em Saúde (Pró-Saúde) que surgiu numa parceria do M inistério da Saúde (M S) com o da E ducação (M EC), a partir da Secretaria de G estão do T rabal ho e da E ducação Superior (SG T ES), da E ducação Superior (SE SU ) e com 0 apoio do Instituto Nacional de Estudos e Pesquisas E ducacionais A nísio T eixeira (I NEP) ${ }^{(6,7)}$.

Esse projeto, lançado em novembro de 2005 pela Portaria Interministerial M S/ M E C no 2101, reconhece 0 processo de formação em saúde como um componente para a qualificação da força de trabalho e para a efetivação da política nacional de saúde. Assim, era importante refletir a formação em saúde, sendo o desafio favorecer a articulação entre as instituições formadoras e os serviços ${ }^{(6)}$.

É objetivo do Pró-Saúde promover a integração ensino/ serviço para a reorientação da formação profissional numa abordagem integral e contextualizada do processo saúde/ doença. Esta proposta enfatiza ainda a atenção básica e a promoção de transformações nos processos de geração de conhecimentos, relacionando as necessidades sociais às dimensões históricas, econômicas e culturais dos distintos grupos ${ }^{(6,7)}$.

A pós a elaboração do programa pelas instituições parceiras supracitadas foi lançado, pelo $\mathrm{M}$ inistério da Saúde, o edital SGTES/ M S no 1, mesmo ano e data da portaria supracitada, com a concorrência pública do Pró-Saúde 1. E ste edital objetivava prover de recursos financeiros as instituições de formação em saúde, com o intuito de contribuir com a reorientação do ensino a partir da articulação com os serviços. A Faculdade de Enfermagem (FAEN) da U niversidade do Estado do R io G rande do N orte (UER N) submeteu sua proposta de reorientação da formação em enfer magem, tendo o projeto sido aprovado.

As atividades desenvolvidas foram desde a re-estruturação dos espaços institucionais, para acomodar a dinâmica funcional do serviço, até a reflexão das práticas de ensino/ aprendizagem, articulando a dimensão teórica do conhecimento com a vivência prática nos espaços das ações em saúde. N este sentido, se buscou fortalecer a parceria institucional academia e serviço para, em conjunto, se contribuir com a transformação dos saberes/ fazeres cotidianos.

A tualmente estamos vivenciando o segundo ano das atividades propostas no projeto original 
e, numa observação preliminar, verificamos que aspectos relacionados à articulação teoria/ prática e a produção coletiva em saúde ainda precisam ser melhorados.

Assim, consideramos importante fazer uma descrição da implantação e impacto deste projeto nas atividades desenvolvidas nos cenários de ensino e trabal ho, pois é necessário refletir o processo de formação em enfermagem e a articulação ensino/ serviço, conhecendo as ideias dos trabal hadores dos serviços quanto às mudanças possíveis.

Diante do exposto, este estudo, fruto de um projeto de iniciação científica que culminou com a produção de uma monografia(8), objetivou conhecer a implantação e inserção do Pró-Saúde no município de M ossoró, Rio Grande do N orte, para entender como os atores da atenção básica se posicionam diante da articulação ensino/ serviço.

\section{MET ODOLOGIA}

Para refletir a formação em saúde e a articulação ensino/ serviço a partir da implantação do Pró-Saúde foi realizada uma criteriosa pesquisa bibliográfica, para uma apropriação da temática, e uma investigação de natureza qualitativa. A abordagem qualitativa melhor se aplicou a este processo por favorecer o caráter participativo dos pesquisadores, possibilitando um olhar diferenciado para os discursos que emergem ${ }^{(9)}$. Sendo a produção do conhecimento é um processo interacional, essa metodologia contribui para o repensar crítico e reflexivo acerca de concepções e práticas que embasam o processo de formação do enfermeiro na U niversidade do E stado do R io G rande do Norte.

0 lócus deste estudo foram as U nidades Básicas de Saúde (U BS) do Bairro Liberdade II, Santo Antônio e Lagoa do M ato em M ossoró, Rio Grande do N orte, parceiras da FAEN na instalação e continuidade do Pró-Saúde. N estas instituições, local izadas em bairros periféricos do município que vivenciam uma série de problemas estruturais e cujos moradores são, em sua maioria, considerados de baixa renda, atuam oito equipes de Saúde da Família.

Para a coleta dos dados foi elaborado um roteiro de entrevista com questões relacionadas ao conhecimento do projeto, implantação, articulação ensino/ serviço e mudanças estruturais e funcionais na dinâmica de atenção à saúde. Este roteiro foi pré-testado e após mínimas alterações, considerado adequado para os objetivos deste estudo.
Os critérios para compor a amostra foram a participação nas atividades propostas pelo PróSaúde, a colaboração com a pesquisa concedendo a entrevista e a assinatura do T er mo de Consentimento L ivre e E sclarecido (T CLE ). As entrevistas gravadas, num total de seis, foram transcritas de forma fidedigna e os participantes identificados a partir de pseudônimos.

A pós sucessivas leituras do material, foi utilizada como técnica para análise dos dados o método de análise de conversação, pois este discute a interação social e os aspectos que interatuam na produção social das conversas cotidianas ${ }^{(10)}$. No caso em destaque estudou-se o Pró-Saúde nos espaços das U nidades Básicas de Saúde (UBS) de M ossoró procurando entender como os atores sociais se percebem nesta dinâmica. Para este artigo, em particular, foram construídas categorias que demonstraram a implantação do Pró-Saúde na perspectiva dos colaboradores deste estudo.

Este projeto foi submetido ao Comitê de Ética em Pesquisa (CEP) da UERN, sendo respeitados os aspectos éticos das pesquisas com seres humanos, especialmente nas questões relacionadas ao anonimato e a privacidade, tendo sido aprovado sob o parecer final $n$ 우 08/ 09 CEP/ UERN.

\section{RESULTADOS E DISCUSSÕES}

A partir dos relatos que emergiram nas entrevistas, foram delimitadas três construções discursivas quanto à implantação e estruturação do Pró-Saúde diante da articulação ensino/ serviço no município de M ossoró.

A primeira construção apresenta os conhecimentos dos enfermeiros quanto à estratégia PróSaúde, trazendo a percepção destes atores acerca do projeto e da sua capacidade de transformação do ensino e da prática cotidiana. A segunda aborda a implantação do Pró-Saúde nas unidades básicas nas quais esses profissionais estavam inseridos, retratando a integração ensino/ serviço. Por fim, a terceira construção discute a capacidade de articulação, na visão dos participantes, entre a academia e o serviço, a partir da estratégia Pró-Saúde.

\section{Acerca do Pró-Saúde}

Para apreender os conhecimentos e práticas diante da estratégia Pró-Saúde, foi solicitado que os sujeitos discorressem sobre seus entendimen- 
tos acerca do Programa N acional de Reorientação da Formação Profissional em Saúde. Alguns profissionais afirmaram tratar-se de:

U m projeto entre o M inistério da $\mathrm{E}$ ducação e as unidades básicas de saúde, mandando recursos humanos e materiais, custeando al guns equi pamentos como máquina fotográfica, computador, coisas que a unidade não tinha (E nfermeira M argarida).

É um programa de extensão da U niversidade, que na verdade é um programa do M inistério da Saúde quea universidade tem responsabilidade junto com as unidades (E nfermeira R osa).

No primeiro relato, o projeto adquire uma dimensão estrutural física e material, sendo os recursos materiais, não existentes na unidade e adquiridos com a parceria, considerados o foco principal do programa. Esta visão demonstra a distância entre o objetivo da transformação a partir da articulação ensino/ serviço para uma dimensão mais tecnológica, pelas vantagens diante de equipamentos. 0 segundo discurso vê a estratégia numa dimensão extensionista, desconsiderando a complexidade da reflexão acerca da reorientação do ensino e da prática cotidiana. Esta visão reducionista parece não articular a extensão univer sitária como uma estratégia para a (re)orientação do processo ensinar/ aprender em saúde ${ }^{(11)}$.

$\mathrm{N}$ os discursos de alguns atores, evidenciamse justificativas para os mínimos conhecimentos acerca do Pró-Saúde, pautadas na dinâmica cotidiana dos serviços que impossibilita uma maior apropriação com proposta do prog rama do qual são parceiros:

\section{J á li sobre o P ró-Saúde, mas somos tão ocupados nas unidades que não dá para detalhar, mas é muito inte- ressante $\mathrm{N}$ ós lemos 0 manual dizendo como fazer a implantação dos serviços e nos ensinando, facilitando 0 desenvolvimento das atividades (E nfermeira Rosa).}

Este relato demonstra o parco conhecimento da responsabilidade que é pactuada pelas instituições quanto à proposta do Pró-Saúde e a precária reflexão acerca da dimensão política institucional. Percebe-se que o conhecimento sobre 0 projeto é fragmentado, o que implica na dificuldade de operacionalização diante das mudanças propostas para a reflexão da formação e da assistência em saúde.
De fato, é necessário uma maior sensibilização das pessoas que pactuam o projeto para a reflexão do ensino e das práticas em saúde/ enfermagem, sendo a sensibilização e a capacitação permanente dos trabal hadores inseridos na atenção à saúde situação emergente ${ }^{(11)}$. É importante que academia e serviço se percebam como parceiros, numa relação dialógica, propondo a realização de atividades que reflitam e transformem as ações em saúde ${ }^{(12)}$.

Para mudar é necessário conhecer, discutir, repensar e reorientar. 0 conhecimento de um contexto social, espaço onde se insere as necessidades de saúde, desvela a problemática e a aplicabilidade dos saberes produzidos nos espaços institucionais, tendendo a ajudar a reflexão entre o ideal e o possível para a saúde. Assim, para se pensar em transformar as práticas vigentes é preciso se apropriar da realidade concreta que demonstra as necessidades sociais e de saúde da população, refletindo a luz dos ideais e propondo um novo, que deve ser incorporado e reproduzido por todos os envolvidos.

\section{Implantando o Pró-Saúde}

A implantação do Pró-Saúde nos serviços de M ossoró aconteceu após aprovação em reunião do Consel ho Administrativo e Diretor (CON SAD) da FAEN, tendo início a sensibilização dos trabaIhadores das equipes de saúde da família das unidades definidas. Para alguns, a implantação foi lenta e articulada:

O uvimos falar sobre o Pró-Saúde e eu já tinha visto algumas coisas na inter net, mas ainda não sabia ao cer to 0 que era. Depois soubemos que vinha para M ossoró [ .... . E ntão para iniciar o P ró-Saúde houve reuniões. $\mathrm{N}$ elas desenvolvemos protocolos, sala de situação, discutimos também a capacitação dos funcionários, de que maneira inserir mais os usuários nos serviços (E nfermeira Perpétua).

Neste relato, fica evidente que os atores dos serviços se envolveram, participando da definição das estratégias para a reflexão do ensino e da prática cotidiana. Já outro discurso apresenta uma dimensão difer enciada deste contexto:

$\mathrm{N}$ a realidade eu não sei direito como foi a inserção do P ró-Saúde nos ser viços, mas sempre tinha estratégias de ação; sempre tinha um projeto que eles nos apresen- 
tavam e a gente botava em ação na área (E nfermeira Bugari).

É possível referendar que para a transformação do ensino e da prática é preciso articulação na construção das parcerias nestes espaços para que ações e saberes sejam produzidos conjuntamente, numa melhor aproximação com as necessidades da população(5,13). E mbora o relato inicial traga a produção/ organização coletiva das ações propostas, na sequência, evidencia-se a passividade dos serviços diante da academia enquanto produtora do saber a ser reproduzido no cotidiano. Esta possível dificuldade na construção coletiva pode favorecer um olhar não reflexivo para a dinamicidade da saúde nos diferentes contextos, necessidades e possibilidades.

N esta dimensão o trabalhador parece colocar em prática o proposto pela academia numa tendência a reproduzir as ações mecanicamente. É possível inferir que quando uma das partes é condutora do processo, mais comumente a U niversidade pelo seu caráter formador, é possível a construção de um projeto verticalizado, que não contextualiza os serviços, tornando-se falho na sua capacidade de transformação. A prática reflexiva favoreceria a aproximação com a realidade, podendo gerar um melhor enfrentamento das necessidades da populaçãa(3).

Além disso, a dinâmica atual na atenção em saúde/ enfer magem, articulada ao Pró-Saúde, aponta para uma formação crítica, reflexiva, com competências específicas para a co-gestão de novos espaços de prática e para a autonomia dos diferentes atores ${ }^{(7,14)}$

Todavia, mesmo apontando para a articulação academia/ serviços de saúde, há questões estruturais que limitam este processo, sendo a rotatividade dos trabalhadores nas unidades um destes aspectos:

Quando cheguei já estava implantado, eu não sei informar sobre a implantação, pois faz menos de um ano e meio que eu cheguei e da lá para cá não me disseram nada. E ntão, as dificuldades para implantação não sei bem dizer [ ...] (Enfermeira M argarida).

A rotatividade dos profissionais nas unidades de saúde é algo que tende a acarretar prejuízos nas práticas e na vinculação com as famílias residentes na área. Estas dificuldades também se expressam na dinâmica do Pró-Saúde, pois quem chega tende a se sentir despreparado para entender e vivenciar as idéias em andamento. U ma maior aproximação entre a universidade e os serviços poderia torná-los co-responsáveis com a produção em saúde.

Todo caso, é válido refletir que a integração ensino/ serviço deve se caracterizar como uma via de mão dupla, na qual um e outro seriam parceiros na apropriação e reprodução das ações organizadas. Contudo, no processo de formação em saúde ainda vigente é comum a espera de tudo pronto a partir da academia, desconsiderando-se que o conhecer é contínuo, não sendo o diploma suficiente para garantir a qualificação esper ada para os avanços científicos e tecnológicos presentes nestes espaços $^{(3)}$. 0 ensino em saúde ainda tende a ter um formato centrado em conteúdos e na pedagogia tradicional, não havendo articulação ensino, pesquisa e extensão e nem uma orientação integrada entre saber e fazer para que a formação potencial ize competências para a integ ral idade ${ }^{(3,15)}$.

Por assim estarem condicionados, alguns profissionais acomodam-se diante do produto e as interseções entre instituições de ensino superior e unidades de saúde tendem a ser limitadas, não sendo possível articular saber/fazer com a realidade concreta. Esta dinâmica reforça o modelo de formação em saúde, no qual o conhecimento e as ações são repassadas para os serviços não havendo construção coletiva que favoreça a problematização do contexto e a reflexão do cotidiano. A caso houvesse esta interação, o risco de se consider ar a universidade como referência do saber legítimo estaria diminuído e os serviços de saúde ganhariam destaque como espaço de aprendizagem e produtor de conhecimento(16).

A importância da construção coletiva está na participação e na troca de opiniões/ conhecimentos, evitando passividade e a exclusiva reprodução das propostas o que contribuiria para a conformação de estratégias que articulassem todas as partes envolvidas na dinâmica do cuidar.

Há que se ressaltar a responsabilidade da academia na formação, englobando aspectos de produção de subjetividade, habilidades, técnicas e pensamentos e 0 adequado conhecimento do SUS. $\mathrm{N}$ este processo o objetivo seria a transformação das práticas e da organização do trabalho, que devem estar estruturadas pela problematização do contexto e fazeres cotidianos ${ }^{(17)}$. Então, o estímulo às re- 
flexões críticas entre docentes, profissionais e estudantes inseridos nos diversos cenários de aprendizagem, mostraria a necessidade de se conhecer a realidade para refletir, sintetizar e propor aspectos norteadores da formação em saúde ${ }^{(16)}$.

\section{A articulação ensino/ serviço: o olhar de quem cuida}

N a proposta de ação sugerida pelo Pró-Saúde é preciso que haja uma contínua e reflexiva articulação entre academia e os serviços de saúde para que o produto final, em ambos, contribua para a reorientação do ensino e das práticas cotidianas. Diante disto, questionou-se aos enfermeiros parceiros do projeto Pró-Saúde sobre a articulação ensino/ serviço. As respostas apontaram para:

Existem algumas coisas que acontecem que nós não somos consultados [ os profissionais das unidades]. A lgumas coisas que a gente tem que engolir e pronto! A gente deveria ter mais participação em relação às questões do estágio supervisionado [ ...] . P orquea unidade deveria antes de planejar, antes mesmo de definir o horário das disciplinas desses alunos, sermos consultadas para articular horário e ações existentes [ ... . . N ós somos convidados para eventos e algumas reuniões da universidade, mas vamos como convidados (E nfermeira Perpétua).

H á sim articulação entre academia e serviços. E les sempre estão nos visitando procurando saber se estamos precisando de alguma coisa, sempre participando das reuniões [ ...] . A universidade trouxe alguns projetos e a gente colocou em prática dentro dos nossos grupos e na área, por exemplo, sobre o fumo, alimentação, ex ercícios físicos, procura dos pacientes com diabetes e dos comunicantes, busca ativa. Tudo isso foi importante (Enfermeira Buriti).

D essas falas é possível inferir que existem lacunas nessa articulação, sendo mais frequente as ações verticais para o repasse de informações e atividades a serem desenvolvidas. E mbora seja rotina durante as práticas propostas pela FAEN/ UERN a construção de um projeto de intervenção, a partir das necessidades evidenciadas na rotina cotidiana, parece ainda persistir a desarticulação entre a proposta e o contexto do serviço. E sta dinâmica interfere na capacidade individual e institucional de reinterpretar a realidade, modificando as ações corriqueiras. A pesar dos enfermeiros dos serviços serem caracterizados como par- ceiros, a academia ainda tende a produzir suas propostas de modo isolado, chegando às unidades de saúde com tudo já organizado.

Sabe-se que há a necessidade de se investir na organização do processo ensino-aprendizagem de modo que possa proporcionar ao aluno/ profissional de saúde mais do que capacidades técnicas ${ }^{(13)}$. D essa forma o ensino acadêmico deveria contribuir para tornar o futuro trabalhador um agente ativo das mudanças necessárias para a construção de um país mais equânime e justo, beneficiando a população assistida por estas pessoas ${ }^{(1)}$.

Portanto, a articulação ensino/ serviço deveria se caracterizar como eixo norteador do processo de formação e do trabalho em saúde. Esta dinâmica favoreceria a indissociabilidade, proporcionando experiências singulares e possibilitando 0 trabal ho interdisciplinar e multiprofissional. Neste processo, os futuros profissionais construiriam sua prática pautada em um cuidado não fragmentado e na percepção do usuário enquanto cidadão.

É provável que esta articulação ensino/ serviço, que integra teoria e prática, seja possível a médio e/ ou longo prazo, a partir do processo já delineado no interior de al guns espaços de produção da saúde. N este tempo seria possibilitado ao aluno a reflexão da realidade, a el aboração de críticas e a busca por soluções adequadas para os problemas de saúde ${ }^{(13)}$, o que favoreceria a indissociabilidade entre o processo de formação e o contexto das práticas cotidianas.

\section{CONCLUSÕES}

A Faculdade de Enfermagem há muito tempo tenta estabelecer o diálogo com as diversas unidades de saúde no município de M ossoró no sentido de promover a possível articulação ensino/ serviço. Entretanto, mesmo com todos os avanços nesta discussão, ainda há diversas lacunas a serem refletidas. Assim, o Pró-Saúde surgiu como uma estratégia que tornava possível a discussão de aspectos que contribuiriam para a reorganização da formação profissional tendo em vista as demandas emergentes na comunidade e as políticas de saúde propostas pelo E stado.

Todavia os problemas ainda persistem, mesmo nos espaços parceiros desta dinâmica. Há o distanciamento entre os pares, pela dificuldade 
em se desconstruir a formação tradicional em saúde, com seu enfoque predominantemente biologicista e curativo, e que não capacita para a promoção da saúde, não contribuindo com as propostas e políticas de saúde destinadas a população(4).

De fato, é sabido que para aproximar a formação profissional das necessidades de saúde da população é necessário superar o paradigma "conteudista" predominante, que leva a uma lógica linear de produção de conhecimento centrado na academia enquanto produtora deste saber ${ }^{(12)}$.

Já a universidade parece estar um tanto ausente na coresponsabilização dos distintos atores na produção de propostas coletivas para o processo de formação em saúde. Por este distanciamento, há uma tendência em não se atender aos interesses da formação crítica e reflexiva, sendo ainda incipiente a transformação na assistência e na construção de conhecimento. M udanças desta ordem contribuiriam para a promoção da saúde, uma das vertentes preconizadas pelo SUS, possibilitando a intervenção nos determinantes do processo saúde doença. É função da universidade a formação de generalistas, e para que isso venha a ocorrer com a qualidade necessária a inserção precoce do discente/ profissional no mundo do trabalho. Assim, haveria uma tendência para uma atuação crítica e reflexiva, numa visão global, integrada e que tenha como eixo central a promoção da saú$\mathrm{de}^{(4,12)}$

Para que o Pró-Saúde venha a se configurar enquanto um projeto de reorientação do ensino a partir da prática ${ }^{(7)}$, é necessário o interesse no desenvolvimento de suas ideias por parte de todos os envolvidos (profissionais, alunos e universidade). Assim, se estabel eceria uma articulação entre estes importantes atores o que minimizaria os entraves que vão surgindo na sua operacionalização.

Por se entender que o conhecer e transformar precisa de sucessivas aproximações, reflexões e avaliações, é imprescindível apreender que trabalhos deste porte precisam ser revistos em suas potencialidades e limitações. Ao se delinear estratégias desta magnitude, de modo efetivo, estar-se-ia contribuindo com a transformação das práticas corriqueiras, com reflexos na formação em saúde. De fato, é preciso rever e, se necessário, reorientar a proposta delineada pelo Pró-Saúde FAEN/ UERN, a partir da aplicabilidade na di- nâmica cotidiana dos serviços, reconhecendo os aspectos que interatuam na transformação da produção dos saberes e práticas tendo em vista as necessidades da população assistida pelo SU S.

\section{REFERÊ NCIAS}

1 Ceccim R B. A emergência da educação e ensino da saúde: inter seções e intersetorialidades. Rev Ciênc Saúde. 2008;1(1):9-23.

$2 \mathrm{M}$ ariotti H. As paixões do ego: complexidade, política e solidariedade. São Paulo: Palas Athena; 2000.

3 Campos G W S. T ratado de saúde coletiva. São Paulo: Fiocruz; 2007.

4 Chiesa M A, Nascimento GDD, Bracially DAL, Oliveira CA M , Coampone T H M . A formação de profissionais da saúde: aprendizagem significativa à luz da promoção da saúde. CogitareE nferm. 2007;12(2):23640.

5 Albuquerque VS, Gomes AP, Rezendell CH A, Sampaio M X, D ias OV, L ugarinho R M . A integração ensino-serviço no contexto dos processos de mudança na formação superior dos profissionais da saúde. Rev Bras E duc M éd. 2008;32(3):356-62.

6 M inistério da Saúde (BR), Secretaria Executiva. PróSaúde: Programa Nacional de Reorientação da Formação Profissional em Saúde. Brasília (DF); 2005.

7 M inistério da Saúde (BR ), Secretaria Executiva. Programa Nacional de Reorientação da Formação Profissional em Saúde: objetivos, implementação e desenvolvimento potencial. Brasília (D F); 2007.

8 L eite ID R. 0 Pró-Saúde e a reorientação do ensino e da prática [ monografia] . M ossoró: F aculdade de E nfermagem, U niversidade do Estado do Rio Grande do N orte; 2010.

9 G onzález Rey FL. Pesquisa qual itativa em psicologia: caminhos e desafios. São Paulo: Pioneira Thomson Learning; 2002.

10 Flick U. Introdução à pesquisa qualitativa. $3^{\text {nd }}$ ed. Porto Alegre: Artmed; 2009.

11 Lopes SR S, Piovesan ETA, M elo LO, Pereira M F. Potencialidades da educação permanente para a transformação das práticas de saúde. Comun Ciênc Saúde. 2007;18(2):147-55 
12 M orita M C, Kriger $L, G$ asparetto $A, T$ anaka $E E$, Higasi MS, M esas $A E$, et al. Projeto Pró-Saúde Odontologia: relato de atividades iniciais em universidades do Estado do Paraná. Espaç Saúde. 2007; 8(2):53-7.

13 Pereira JG, F racolli LA. A contribuição da articulação ensino-serviço para a construção da vigilância da saúde: a perspectiva dos docentes. Rev LatinoA m Enfermagem. 2009;17(2):167-73.

14 Backes DS, E rdmann AL. Formação do enfermeiro pelo ol har do empreendedorismo social. Rev G aúcha Enferm. 2009;30(2):242-8.
15 Claus SM . Competências para o processo de gerenciamento da atenção básica: contribuição para a formação e a atuação de profissionais da saúde [ tese] . Campinas: Faculdade de Ciências M édicas, U niversidade E stadual de Campinas; 2005.

16 A raújo D, M iranda GCM , Brasil LS. Formação de profissionais de saúde na perspectiva da integralidade. Rev Baiana Saúde Pública. 2007;31(1):2031.

17 Ceccim RB, Feuerwerk M CL. 0 quadrilátero da formação para a área da saúde: ensino, gestão, atenção e controle social. Physis. 2004;14(1):41-65.
Endereço da autora / D irección del autor / Author's address:

F átima Raquel Rosado M orais

Rua Dionísio F ilgueira, 383, Centro

59610-090, M ossoró, R N

E-mail: frrm@bol.com.br
Recebido em: 06/ 06/ 2010

A provado em: 18/ 08/ 2010 